\title{
Fibronectin Induces Pro-MMP-2 Activation and Enhances Invasion in H-Ras-Transformed Human Breast Epithelial Cells
}

\author{
Jong-Sook KIM, and Aree MooN* \\ College of Pharmacy, Duksung Women's University, Seoul 132-714, Republic of Korea
}

(Received June 9, 2009; Revised June 22, 2009; Accepted June 17, 2009)

\begin{abstract}
Interactions between tumor cells and the extracellular matrix (ECM) strongly influence tumor development, affecting cell survival, proliferation and migration. Fibronectin, a major component of ECM, has been shown to interact with integrins especially the $\alpha 5 \beta 1$ integrin. Cell invasion and metastasis are often associated with matrix metalloproteinases (MMPs) which are capable of digesting the different components of the ECM and basement membrane. MMP-2 is produced as a latent pro-MMP-2 (72 kDa) to be activated, resulting the $62 \mathrm{kDa}$ active MMP-2. In this study, we investigated the effect of fibronectin on activation of pro-MMP-2 and the cellular invasiveness in H-Ras-transformed MCF10A human breast epithelial cells. Here we show that fibronectin induces activation of pro-MMP-2 and up-regulation of MT1-MMP and TIMP-2 in H-Ras MCF10A cells. These results demonstrate that H-Ras MCF10A cells secrete high levels of active MMP-2 when cultured with fibronectin, suggesting a possible interaction between the ECM network and $\mathrm{H}$-Ras MCF10A cells to generate active MMP-2 which is important for proteolysis and ECM remodeling. Invasive and migratory abilities of $\mathrm{H}$-Ras MCF10A cells were enhanced by fibronectin. Fibronectin up-regulated the expression of $\beta 1$ integrin which may play a role in cellular responses exerted by fibronectin. Since acquisition of pro-MMP-2 activation can be associated with increased malignant progression, this study provides a mechanism for the cell surface-matrix degrading effect of fibronectin which will be crucial to breast cell invasion and migration.
\end{abstract}

Keywords: Fibronectin, Active MMP-2, H-Ras MCF10A, Breast cell invasion

\section{INTRODUCTION}

Interactions between the tumor cell and the extracellular matrix (ECM) strongly influence tumor growth, cell survival, proliferation, migration and invasion (Juliano, 2002). During cancer development, the ECM of the tissue in which the tumor grows is extensively remodeled both by degradation of preexisting ECM molecules and by the neosynthesis of ECM components (Kaspar et al., 2006). Cell adhesive interactions with ECM proteins such as fibronectin are mediated by specific cell surface receptors, called integrins. Fibronectin is a multifunctional extracellular glycoprotein which has at least two independent cell adhesion sites with different receptor specificities (Akiyama et al., 1995). One of the principal fibronectin binding integrins is integrin $\alpha 5 \beta 1$, which interacts with the

${ }^{*}$ Corresponding author

Tel: +82-2-901-8394 Fax: +82-2-901-8386

E-mail: armoon@duksung.ac.kr central cell adhesive region of fibronectin for maximal binding (Akiyama et al., 1995).

The process of tumor cell invasion and metastasis requires degradation of connective tissue associated with basement membrane and interstitial connective tissue (Stetler-Stevenson et al., 1993; Yu et al., 1996). Matrix metalloproteinases (MMPs), a family of zinc dependent endopeptidases, are capable of digesting the components of the ECM and basement membrane. Mounting evidence strongly implicates MMPs, particularly MMP-2, in the process of tumor, including a positive correlation between MMP-2 expression and invasive potential (Stetler-Stevenson et al., 1993; Curran and Murray et al., 1999; Kleiner and Stetler-Stevenson et al., 1999).

MMP-2 is produced as a latent pro-MMP-2 (72 kDa) requiring activation for catalytic activity, a process that is accomplished by proteolytic removal of the propeptide domain, resulting the $62 \mathrm{kDa}$ active MMP-2 (Strongin et al., 1995). It has been demonstrated that pro-MMP-2 forms a 
trimolecular complex with membrane type 1 (MT1)-MMP and tissue inhibitor of metalloproteinase (TIMP)-2, and then to be cleaved by an adjacent MT1-MMP molecule on the cell surface (Strongin et al., 1995; Seiki, 2002; Sato et al., 2005).

Mounting evidence from laboratories including ours suggests unique roles of the Ras family members in tumor aggressiveness of breast cancer (Clair et al., 1987; Watson et al., 1991; Clark and Der, 1995). We previously showed that $\mathrm{H}$-Ras induced invasive and migratory phenotypes in MCF10A human breast epithelial cells and $\mathrm{H}$-Ras-induced invasive phenotype was associated closely with the expression of MMP-2 (Moon et al., 2000; Kim et al., 2003; Song et al., 2006). Active form of MMP-2 (62 kDa), however, was not detected in H-Ras MCF10A cells when cultured in polystyrene dishes (Moon et al., 2000). We have recently demonstrated that $\mathrm{H}$-Ras induces pro-MMP-2 activation in MCF10A cells when cultured in two-dimensional gel of type I collagen (Kim et al., 2007). Since fibronectin has been shown to be associated with cellular invasiveness in various cancer cells including breast carcinoma, papillary thyroid carcinoma and colon cancer cells (Arihiro et al., 1993; Ryu et al., 1999; Ito et al., 2004; Ding et al., 2008), the present study investigates the effect of fibronectin on pro-MMP-2 activation in H-Ras MCF10A cells. Here we show that fibronectin induces activation of pro-MMP-2 and invasive and migratory abilities of $\mathrm{H}$-Ras MCF10A cells.

\section{MATERIALS AND METHODS}

\section{Cell culture}

Establishment and culture condition of H-Ras MCF10A cells were described previously (Moon et al., 2000). The cells were cultured in DMEM/F12 medium supplemented with $5 \%$ horse serum, $0.5 \mu \mathrm{g} / \mathrm{ml}$ hydrocortisone, $10 \mu \mathrm{g} / \mathrm{ml}$ insulin, $20 \mathrm{ng} / \mathrm{ml}$ epidermal growth factor, $0.1 \mathrm{mg} / \mathrm{ml}$ cholera toxin, $2 \mathrm{mM} \mathrm{L-glutamine}$ and $0.5 \mu \mathrm{g} / \mathrm{ml}$ amphotericin $\mathrm{B}$ and 100 unit $/ \mathrm{ml}$ penicillin-streptomycin.

\section{In vitro invasion assay}

In vitro invasion assay was performed using 24-well transwell as previously described (Kim et al., 2003). The lower side of the filter was coated with type I collagen, and the upper side was coated with fibronectin $(5 \mu \mathrm{g} / \mathrm{ml})$. Lower compartment was filled with serum-free media containing $0.1 \%$ BSA. Cells were placed in the upper part of the Transwell plate, incubated for $17 \mathrm{~h}$, fixed with methanol and stained with hematoxylin for $10 \mathrm{~min}$ followed briefly by eosin. The invasive phenotypes were determined by counting the cells that migrated to the lower side of the filter with microscopy at $\times \mathbf{4 0 0}$. Thirteen fields were counted for each filter, and each sample was assayed in triplicate.

\section{Transwell migration assay}

In vitro Transwell migration assay was performed using a 24-well transwell unit with polycarbonate filters as previously described (Kim et al., 2003). The lower side of the filter was coated with type I collagen (Sigma Chemical Co). Lower compartment was filled with serum-free media containing $0.1 \%$ BSA. Soluble fibronectin and $2.5 \times 10^{4}$ cells were placed in the upper part of the transwell were plate, incubated for $17 \mathrm{~h}$, fixed with methanol and stained with hematoxylin for $10 \mathrm{~min}$ followed briefly by eosin. The migrative phenotypes were determined by counting the cells that migrated to the lower side of the filter with microscopy at $\times 400$. Thirteen fields were counted for each filter and each sample was assayed in triplicate.

\section{Gelatin zymography}

Cells were cultured in serum-free DMEM/F12 medium containing soluble fibronectin. Conditioned medium was collected and centrifuged at 3,000 rpm for $10 \mathrm{~min}$ to remove cell debris. Gelatinolytic activity of the conditioned medium was determined by gelatin zymogram assay as previously described (Moon et al., 2000; Kim et al., 2003). Areas of gelatinase activity were detected as clear bands against the blue-stained gelatin background.

\section{Immunoblot analysis}

Immunoblot analysis was performed as previously described (Kim et al., 2003). Anti-MT1-MMP, anti-TIMP-2, anti- $\alpha 5$ and anti- $\beta 1$ antibodies were purchased from R\&D Systems Inc. Enhanced chemiluminescence (ECL, Amersham-Pharmacia) system was used for detection. Relative band intensities were determined by quantitation of each band with an Image Analyzer (Vilber Lourmet, France).

\section{RESULTS}

\section{Fibronectin activates pro-MMP-2 in H-Ras MCF10A cells}

To examine the effect of fibronectin on the activation of MMPs, conditioned media of H-Ras MCF10A cells treated with fibronectin were subjected with gelatin zymogram assay. As shown in Fig. 1, an active form of MMP-2 (62 $\mathrm{kDa}$ ) was detected when the cells were treated with $5 \mu \mathrm{g} /$ $\mathrm{ml}$ of fibronectin for $24 \mathrm{~h}$. The results demonstrate that fibronectin induces activation of pro-MMP-2 in H-Ras MCF10A cells. 


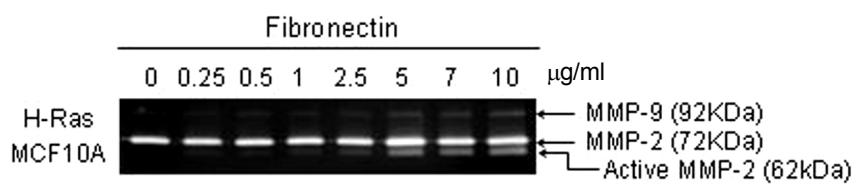

Fig. 1. Fibronectin induces pro-MMP-2 activation in $\mathrm{H}$-Ras MCF10A cells. Gelatin zymogram assay was performed on the conditioned media cultured with the indicated concentration of fibronectin for $24 \mathrm{~h}$. Gelatinolytic activities of secreted MMP-2 and MMP-9 were determined by gelatin zymogram assay.
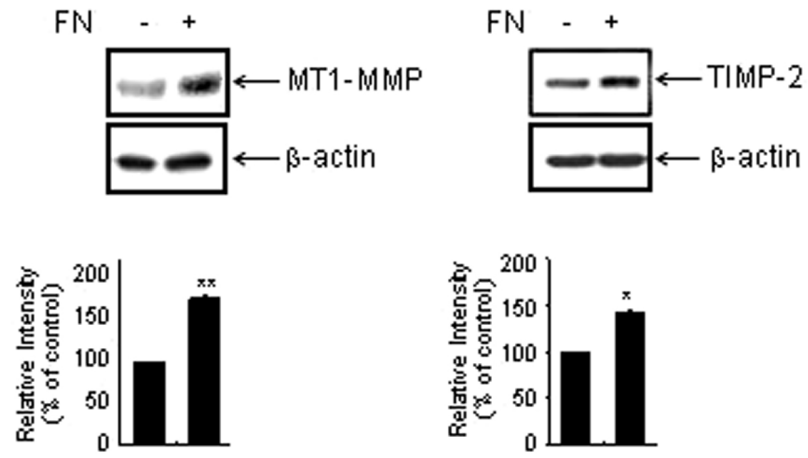

Fig. 2. Fibronectin up-regulates MT1-MMP and TIMP-2 in $\mathrm{H}$-Ras MCF10A cells. Cell lysates were prepared from the cells cultured on $5 \mu \mathrm{g} / \mathrm{ml}$ fibronectin and analyzed for the expression of MT1-MMP ( $57 \mathrm{KDa})$ and TIMP-2 $(25 \mathrm{KDa})$ by immunoblot analysis. $\beta$-actin was used as a loading control. The immunoblots shown are representative of three blots from independent experiments. Relative band intensities from three independent blots were determined by using an Image analyzer. * ${ }^{* *}$ Statistically different from control at $p<0.05$ and $p<0.01$, respectively.

\section{MT1-MMP and TIMP-2 are up-regulated by fibronectin}

It has been reported that MT1-MMP and TIMP-2 form a 'receptor' complex that binds pro-MMP-2, resulting in the proteolysis of bound MMP-2 by an adjacent free MT1-MMP (Itoh et al., 2001). We investigated the effect of fibronectin on the expressions of MT1-MMP and TIMP-2 in H-Ras MCF10A cells. Immunoblot analyses to detect MT1-MMP and TIMP-2 were performed on cell lysates of $\mathrm{H}$-Ras MCF10A cells treated with fibronectin. As shown in Fig. 2, significant increases in MT1-MMP and TIMP-2 were observed in $\mathrm{H}-\mathrm{Ras}$ MCF10A cells treated with $5 \mu \mathrm{g} / \mathrm{ml}$ of fibronectin for $24 \mathrm{~h}$. These data suggest that MT1-MMP and TIMP-2 may be involved in the activation of pro- MMP-2 induced by fibronectin.

\section{Invasion and migration of H-Ras MCF10A cells were increased by fibronectin}

Since MMPs play important roles in cellular invasive and
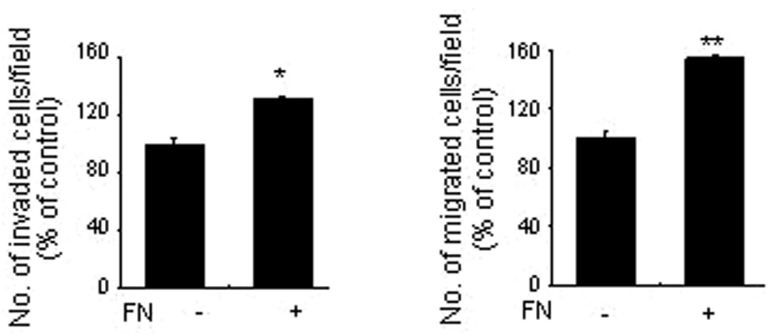

Fig. 3. Fibronectin enhances invasive and migratory phenotypes of H-Ras MCF10A cells. The number of invaded or migrated cells per field was counted $(\times 400)$ in thirteen arbitrary visual fields. The results represent means+SE of triplicates. *, ** Statistically different from control at $p<0.05$ and $p<0.01$, respectively.
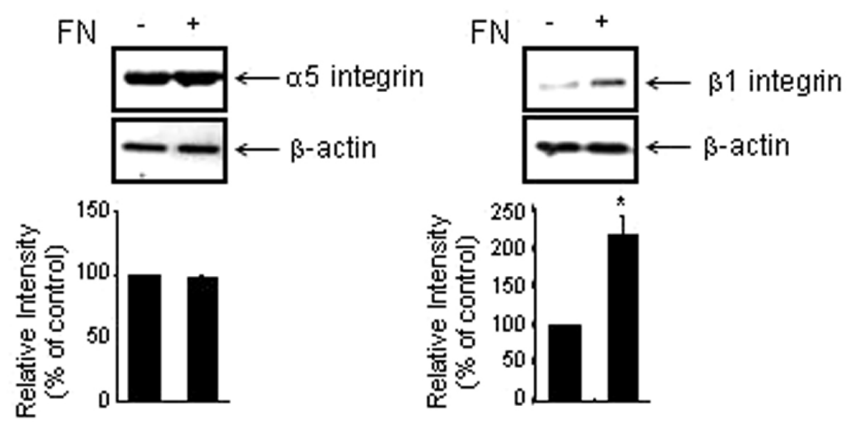

Fig. 4. Fibronectin up-regulates integrin $\beta 1$ in $\mathrm{H}-$ Ras MCF10A cells. Cells were cultured on $5 \mu \mathrm{g} / \mathrm{ml}$ fibronectin and analyzed for the expression of $\alpha 5$ and $\beta 1$ integrins by immunoblot analysis. $\beta$-actin was used as a loading control. The immunoblots shown are representative of three blots from independent experiments. Relative band intensities from three independent blots were determined by using an Image analyzer. *Statistically different from control at $p<0.05$.

migratory phenotypes, we then investigated the effect of fibronectin on H-Ras MCF10A cell invasion and migration. Treatment of H-Ras MCF10A cells with $5 \mu \mathrm{g} / \mathrm{ml}$ of fibronectin for $17 \mathrm{hr}$ caused significant increases in invasive and migratory abilities of H-Ras MCF10A cells (Fig. 3), indicating the enhancing effect of fibronectin on H-Ras-induced invasion and migration of human breast epithelial cells.

\section{Fibronectin induces expression of $\beta 1$ integrin}

Integrins $\alpha 5$ and $\beta 1$ are known to be major receptors for fibronectin. We examined if expression of these integrins was affected by fibronectin by immunoblot analysis. The expression level of $\alpha 5$ integrin was strong in H-Ras MCF10A cells while the basal level of $\beta 1$ integrin was very weak (Fig. 4). Treatment of H-Ras MCF10A cells with $5 \mu \mathrm{g} /$ $\mathrm{ml}$ of fibronectin for $24 \mathrm{~h}$ significantly induced the ex- 
pression of $\beta 1$ integrin whereas the level of $\alpha 5$ integrin was not significantly altered. These results demonstrate that fibronectin up-regulates the expression of $\beta 1$ integrin in $\mathrm{H}$-Ras MCF10A cells which may play a role in cellular responses exerted by fibronectin.

\section{DISCUSSION}

Invasion of the cancer cells into the surrounding matrix is crucial to metastasis (Liotta et al., 1980). An essential part of invasion includes degradation of the ECM components such as type I collagen, fibronectin and laminin and the basement membrane by members of the MMP family (Ura et al., 1989; Fidler, 1990). We have previously shown that type I collagen induces activation of MMP-2 only in the invasive H-Ras MCF10A cells but not in the non-invasive N-Ras MCF10A cells (Kim et al., 2007).

This study investigates the effect of fibronectin on pro-MMP-2 activation in H-Ras MCF10A cells. Here, we show that fibronectin induces activation of pro-MMP-2 and enhances the invasive/migratory phenotypes in H-Ras MCF10A cells. These results suggest that H-Ras MCF10A cells interact with the ECM network to generate active MMP-2 which is important for proteolysis and ECM remodeling.

In consistent with our results, involvement of fibronectin in MMP activation and cell invasion has been demonstrated in various cell systems. Cultivation of cells in the presence of fibronectin results in increased extracellular protease activity and activation of MMP-2/-9 in MCF-7 human breast cancer cells (Munshi and Stack, 2006; Das et al,. 2008). Activation of MMP-2 was also observed in HT-1080 fibrosarcoma cells grown in the presence of fibronectin (Stanton et al., 1998). Fibronectin deposited in the extrcellular matrix of tumors strongly promotes the migration and intracerebral invasion of glioma cells (Enam et al., 1998; Ohnishi et al.,1998).

Proteolytic activation of MMP-2 involves a coordinated interplay of pro-MMP-2, MT1-MMP and TIMP-2 (Goldberg et al., 1989; Hernandez-Barrantes et al., 2000; Wang et al., 2000). Our data showed that the cellular level of MT1-MMP and TIMP-2 increased when H-Ras MCF10A cells were cultured with fibronectin, suggesting the role of MT1-MMP and TIMP-2 in MMP-2 activation of H-Ras MCF10A cells.

Cellular interaction with fibronectin is mediated largely through integrin receptors (Akiyama et al., 1995). The role of $\alpha 5 \beta 1$ integrin and fibronectin interaction in tumor cell invasiveness has been investigated in several studies (Brakebusch et al., 2002; Danen et al., 2002). Our results show that the expression level of $\beta 1$ integrin was increased by fibronectin treatment while $\alpha 5$ integrin level was not significantly altered. Since the basal level of $\alpha 5$ is high and that of $\beta 1$ integrin is very low of H-Ras MCF10A cells, fibronectin treatment may result in a more balanced expression of these integrins. Involvement of other integrins in fibronectin-induced pro-MMP-2 activation is currently under investigation.

In the present study, we demonstrate that fibronectin induces activation of pro-MMP-2 in H-Ras MCF10A cells. These results suggest that in an in vivo environment, $\mathrm{H}$-Ras MCF10A cells may secrete active MMP-2 which is likely to remodel ECM and enhance invasion and migration. Since the activation of pro-MMP-2 can be associated with increased malignant progression, this study provides a mechanism for the cell surface-matrix degrading effect of fibronectin which will be crucial to breast cell invasion and migration.

\section{ACKNOWLEDGMENTS}

This work was supported by the Korea Research Foundation Grant funded by the Korean Government (MOEHRD) (KRF-2008-314-C00230).

\section{REFERENCES}

Akiyama, S. K., Olden, K. and Yamada, K. M. (1995). Fibronectin and integrins in invasion and metastasis. Cancer Metastasis. Rev. 14, 173-189.

Arihiro, K., Inai, K., Kurihara, K., Takeda, S. and Kaneko, M. (1993). Distribution of laminin, type IV collagen and fibronectin in the invasive component of breast carcinoma. Acta. Pathol. Jpn. 43, 758-764.

Brakebusch, C., Bouvard, D., Stanchi, F., Sakai, T. and Fässler, R. (2002). Integrins in invasive growth. J. Clin. Invest. 109, 999-1006.

Clair, T., Miller, W. and Cho-Chung, Y. (1987). Prognostic significance of the expression of the ras protein with a molecular weight of 21,000 by human breast cancer. Cancer Res. 49, 5290-5293.

Clark, G. J. and Der, C. J. (1995). Aberrant function of the Ras signal transduction pathway in human breast cancer. Breast Cancer Res. Treat. 35, 133-144.

Curran, S. and Murray, G. I. (1999). Matrix metalloproteinases in tumour invasion and metastasis. J. Pathol. 189, 300-308.

Danen, E. H. P., Sonneveld, P., Brakebusch, C., Fassler, R. and Sonnenberg, A. (2002). The fibronectin-binding integrins $\alpha 5$ $\beta 1$ and $\alpha v \beta 3$ differentially modulate RhoA-GTP loading, organization of cell matrix adhesions, and fibronectin fibrillogenesis. J. Cell Biol. 159, 1071-1086.

Das, S., Banerji, A., Frei, E. and Chatterjee, A. (2008). Rapid expression and activation of MMP-2 and MMP-9 upon exposure of human breast cancer cells (MCF-7) to fibro nectin 
in serum free medium. Life Sciences 82, 467-476.

Ding, J., Li, D., Wang, X., Wang, C. and Wu, T. (2008). Fibronectin promotes invasiveness and focal adhesion kinase tyrosine phosphorylation of human colon cancer cell. Hepatogastroenterology. 55, 2072-2076.

Enam, S. A., Rosenblum, M. L. and Edvardsen, K. (1998). Role of extracellular matrix in tumor invasion: migration of glioma cells along fibronectin-positive mesenchymal cell processes. Neurosurg. 42, 599-608.

Fidler, I. J. (1990). Critical factors in the biology of human cancer metastasis. Cancer Res. 50, 6130-6138.

Goldberg, G. I., Marmer, B. L., Grant, G. A., Eisen, A. Z., Wilhelm, S. and He, C. S. (1989). Human 72-kilodalton type IV collagenase forms a complex with a tissue inhibitor of metalloproteases designated TIMP-2. Proc. Natl. Acad. Sci. USA. 86, 8207-8211.

Hernandez-Barrantes, S., Toth, M., Bernardo, M. M., Yurkova, M., Gervasi, D. C., Raz, Y., Sang, Q. A. and Fridman, R. (2000). Binding of active (57 kDa) membrane type 1-matrix metalloproteinase (MT1-MMP) to tissue inhibitor of metalloproteinase (TIMP)-2 regulates MT1-MMP processing and pro-MMP-2 activation. J. Biol. Chem. 275, 12080-12089.

Ito, H., Duxbury, M., Benoit, E., Farivar, R. S., Gardner-Thorpe, J., Zinner, M. J., Ashley, S. W. and Whang, E. E. (2004). Fibronectin-induced COX-2 mediates MMP-2 expression and invasiveness of rhabdomyosarcoma. Biochem. Biophys. Res. Commun. 318, 594-600.

Itoh, Y., Takamura, A., Ito, N., Maru, Y., Sato, H., Suenaga, N., Aoki, T. and Seiki, M. (2001). Homophilic complex formation of MT1-MMP facilitates proMMP-2 activation on the cell surface and promotes tumor cell invasion. EMBO J. 20, 4782-4793.

Juliano, R. L. (2002). Signal transduction by cell adhesion receptors and the cytoskeleton: functions of integrins, cadherins, selectins, and immunoglobulin-superfamily members. Annu. Rev. Pharmacol. Toxicol. 42, 283-323.

Kaspar, M., Zardi, U. and Neri, D. (2006). Fibronectin as target for tumor therapy. Int. J. Cancer. 118, 1331-1339.

Kim, I. Y., Jeong, S. J., Kim, E. S., Kim, S. H. and Moon, A. (2007). Type I collagen-induced pro-MMP-2 activation is differentially regulated by $\mathrm{H}$-Ras and $\mathrm{N}$-Ras in breast epithelial cells. J. Biochen. Mol. Biol. 40, 825-831.

Kim, M. S., Lee, E. J., Choi kim, H. R. and Moon, A. (2003). p38 kinase is a key signaling molecule for $\mathrm{H}$-ras-induced cell motility and invasive phenotype in human breast epithelial cell. Cancer Res. 63, 5454-5461.

Kleiner, D. E. and Stetler-Stevenson, W. G. (1999). Matrix metalloproteinases and metastasis. Cancer Chemother. Pharmacol. 43, 42-51.

Liotta, L. A., Tryggvason, K., Garbisa, S., Hart, J., Foltz, C. M. and Shafie, S. (1980). Metastatic potential correlates with enzymatic degradation of basement membrane collagen. Nature 284, 67-68.

Moon, A., Kim, M. S., Kim, T. G., Kim, S. H., Kim, H. E., Chen, Y. Q. and Choi Kim, H. R. (2000). H-ras, but not N-ras, induces an invasive phenotype in human breast epithelial cells: a role for MMP-2 in the H-ras-induced invasive phenotype. Int. J.
Cancer 85, 176-181.

Munshi, H. and Stack, M. S. (2006). Reciprocal interactions between adhesion receptor signaling and MMP regulation. Cancer Metastasis Rev. 25, 45-56.

Ohnishi, T., Hiraga, S., Izumoto, S., Matsumura, H., Kanemura Y., Arita, N. and Hayakawa, T. (1998). Role of fibronectinstimulated tumor cell migration in glioma invasion in vivo: clinical significance of fibronectin and fibronectin receptor expressed in human glioma tissues. Clin. Exp. Metastasis. 16, 729-741.

Ryu, S., Jimi, S., Eura, Y., Kato, T. and Takebayashi, S. (1999). Strong intracellular and negative peripheral expression of fibronectin in tumor cells contribute to invasion and metastasis in papillary thyroid carcinoma. Cancer Lett. 146, 103-109.

Sato, H., Takino, T. and Miyamori, H. (2005). Roles of membranetype matrix metalloproteinase-1 in tumor invasion and metastasis. Cancer Sci. 96, 212-217.

Seiki, M. (2002). The cell surface: the stage for matrix metalloproteinase regulation of migration. Curr. Opin. Cell Biol. 14, 624-632.

Song, H., Ki, S. H., Kim, S. G. and Moon, A. (2006). Activating transcription factor 2 mediates matrix metalloproteinase-2 transcriptional activation induced by p38 in breast epithelial cells. Cancer Res. 66, 10487-10496.

Stanton, H., Gavrilovic, J., Atkinson, S. J., d'Ortho, M. P., Yamada, K. M., Zardi, L. and Murphy, G. (1998). The activation of proMMP-2 (gelatinase A) by HT-1080 fibrosarcoma cells is promoted by culture on a fibronectin substrate and is concomitant with an increase in processing of MT1-MMP (MMP-14) to a $45 \mathrm{kDa}$ form. J. Cell Science 111, 2789-2798.

Stetler-Stevenson, W. G., Aznavoorian, S. and Liotta, L. A. (1993). Tumor cell interactions with the extracellular matrix during invasion and metastasis. Annu. Rev. Cell Bio. 9, 541573.

Strongin, A. Y., Collier, I., Bannikov, G., Marmer, B. L., Grant, G. A. and Goldberg, G. I. (1995). Mechanism of cell surface activation of $72 \mathrm{kDa}$ type IV collagenase. J. Biol. Chem. 270, $5331-5338$

Ura, H., Bonfil, R. D., Reich, R., Reddel, R., Pfeifer, A., Harris, C. C. and Klein-Szanto, A. J. P. (1989). Expression of type IV collagenase and procollagen genes and its correlation with the tumorigenic, invasive, and metastatic abilities of oncogenetransformed human bronchial epithelial cells. Cancer Res. 49, 4615-4621.

Wang, Z., Juttermann, R. and Soloway, P. D. (2000). TIMP-2 is required for efficient activation of proMMP-2 in vivo. J. Biol. Chem. 275, 26411-26415.

Watson, D. M., Elton, R. A., Jack, W. J., Dixon, J. M., Chetty, U. and Miller, W. R. (1991). The H-ras oncogene product p21 and prognosis in human breast cancer. Breast Cancer Res. Treat. 17, 161-169.

Yu, A. E., Hewitt, R. E., Kleiner, D. E. and Stetler-Stevenson, W. G. (1996). Molecular regulation of cellular invasion-role of gelatinase A and TIMP-2. Biochem. Cell Biol. 74, 823-831. 